\title{
The circumnuclear cold gas environments of the powerful radio galaxies 3C 236 and 4C 31.04
}

\author{
C. Struve and J. E. Conway
}

\author{
Onsala Space Observatory, 43992 Onsala, Sweden \\ e-mail: [christian.struve; john. conway]@chalmers.se
}

Received 2 January 2012 / Accepted 26 July 2012

\begin{abstract}
We present Very Long Baseline Array (VLBA) H I absorption observations of the core regions of the powerful radio galaxies $3 \mathrm{C} 236$ and $4 \mathrm{C} 31.04$. Broad absorption $\left(\Delta v \approx 350 \mathrm{~km} \mathrm{~s}^{-1}\right)$ straddling the systemic velocity is detected in both sources, in each case concentrated on one side of the radio core. We argue that the observations are consistent with the presence of $\mathrm{H}_{\mathrm{I}}$ absorbing disks with $r \approx 900 \mathrm{pc}$ and $r \geq 200 \mathrm{pc}$ in 3C 236 and 4C 31.04 respectively - although given the limited spatial coverage of the HI absorption other interpretations cannot be ruled out. The spatially resolved absorption detected against the counter-jet of $3 \mathrm{C} 236$ shows a velocity gradient with an amplitude and direction supporting a rotating disk model; in contrast only a tentative velocity gradient is seen in the 4C 31.04 data set. While on larger scales both radio galaxies show evidence for interaction and merging we find no direct evidence in either source that cold gas is in-falling onto the radio core. In 3C 236 we instead find against the core blue-shifted absorption that might be part of the fast outflow of neutral gas detected in more sensitive (but lower spatial resolution) observations. Along the main

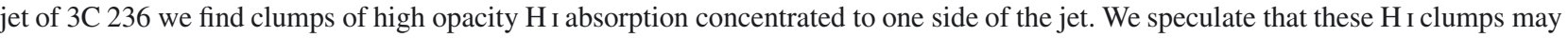
be interacting with the jet, potentially even being the cause of the misalignment of the eastern jet between kiloparsec and megaparsec scales. In addition to its broad absorption 4C 31.04 shows a narrow absorption component $\left(F W H M=23 \mathrm{~km} \mathrm{~s}^{-1}\right)$ redshifted relative to the galaxy systemic velocity by $115 \mathrm{~km} \mathrm{~s}^{-1}$. This feature may be a residual sign of a recent accretion/merging event, however the absorbing gas cloud must be located $\gg 100 \mathrm{pc}$ from the core.
\end{abstract}

Key words. galaxies: active - galaxies: ISM - radio lines: galaxies - galaxies: jets - galaxies: nuclei

\section{Introduction}

The growth of supermassive black holes is known to be dominated by radiatively efficient accretion during "active" phases (Soltan 1982; Marconi et al. 2004; Shankar et al. 2004; Merloni $\&$ Heinz 2008) of gas and dust which reaches the direct vicinity of the black hole (<1 pc; see, e.g., Rees 1984; Magorrian et al. 1998). It has been suggested for a long time that cold gas may play a key role in providing the fuel that triggers active galactic nuclei (AGN) activity but direct observational evidence for its role remains scarce.

On radii of $1-10 \mathrm{pc}$ from the central black hole dust torus structures have been imaged (Jaffe et al. 2004; Tristram et al. 2009; van der Wolk et al. 2010). Modelling of the infrared (IR) spectral energy distributions (SEDs) from AGN heated dust in clumpy tori (Nenkova et al. 2008) provides further evidence for the existence of these structures. Spectral line observations of atomic and molecular species (i.e. H I and $\mathrm{CO}$ ) in absorption provides a potential way to study the kinematics of such small scale gas structures and address the question of whether cold gas is fueling the AGN. Such observations allow us to investigate the morphology and kinematics of the circumnuclear gas structures of radio galaxies from $\mathrm{kpc}$ to pc scales and hence give us clues on how gas reaches the nuclear region.

On kpc scales significant amounts of cold gas (atomic and molecular) have been detected (in emission) in up to $\sim 50 \%$ of early-type galaxies (the hosts of almost all powerful radioloud sources), provided deep observations are made and cluster galaxies are excluded (e.g. Morganti et al. 2006; Combes et al. 2007; Oosterloo et al. 2007, 2010). Typical cold gas masses are $M_{\text {coldgas }} \approx 10^{8} M_{\odot}$, but in individual cases masses can be up to two orders of magnitude larger (see, e.g., Oosterloo et al. 2007). Surveys of the subclass of radio-loud early type galaxies yield similar rates of $\mathrm{H}$ I and $\mathrm{CO}$ detections (e.g. Emonts et al. 2010; Ocaña Flaquer et al. 2010) suggesting no major difference in cold gas mass content between radio-loud and radioquiet early-type galaxies. Differences between radio-loud and radio-quite objects may instead be related to gas kinematics because in order to fuel a black hole gas needs to be transported to the inner few tens of parsec. However clear evidence for inflowing gas in radio loud objects on these scales is still missing. Instead, circumnuclear atomic and molecular disks are detected in a few sources, with typical sizes of tens of pc to $\sim 100 \mathrm{pc}$ (e.g. van Langevelde et al. 2000; Schinnerer et al. 2000; Peck \& Taylor 2001; Morganti et al. 2008; Hicks et al. 2009; Struve \& Conway 2010). Such circumnuclear disks have larger sizes compared to the detected dust torus structures (see above), but they may be continuous with these inner tori and may provide the gas/dust masses necessary to fuel an AGN over its lifetime. However, the relationship between these two structures is far from clear. Moreover, some sources show clearly redshifted absorption components that at least in some cases, must still be located at $100 \mathrm{pc}$ to few kpc distances from the black hole (e.g. Struve \& Conway 2010; Struve et al. 2010).

In order to address the fueling question and to investigate whether cold gas plays an important role in the feeding of the 
torus/black hole it is crucial to study the circumnuclear gas kinematics of powerful radio galaxies at high resolution. For this purpose we have performed NRAO Very Long Baseline Array $\left(\mathrm{VLBA}^{1}\right) \mathrm{H}_{\mathrm{I}}$ absorption observations of two powerful radio galaxies, 3C 236 and 4C 31.04, both of which are known to host large reservoirs of cold gas in their circumnuclear environments. Short reports on the initial data reduction and preliminary results have been presented in Conway $(1996,1997)$ and Conway \& Schilizzi (2000), this present paper presents a fuller re-analysis of the data.

The organisation of this paper is as follows, in Sect. 2 we summarise previous observations of 3C 236 and 4C 31.04 while Sect. 3 describes the new VLBA observations. Section 4 presents the observational results for both sources. A discussion of these results is given in Sect. 5 while Sect. 6 presents our conclusions. At the redshifts of 3C $236(z \approx 0.10)$ and 4 C $31.04(z \approx 0.06)$ for cosmologies with $H_{0}=73 \mathrm{~km} \mathrm{~s}^{-1}$ an angular scale of 100 mas corresponds to approximately $200 \mathrm{pc}$ and 120 pc respectively. All total recession velocities quoted in this paper are heliocentric (optical definition).

\section{Previous observations}

3C 236 is a giant double lobe FR-II type radio galaxy. The outer $4.5 \mathrm{Mpc}$ scale lobes make 3C 236 one of the largest single objects in the universe. The inner $2 \mathrm{kpc}$ sized radio source structure is highly asymmetric with respect to the identified VLBI core (Barthel et al. 1985; Schilizzi et al. 2001) and is misaligned with respect to the outer mega-parsec scale structure by about $7^{\circ}$. It has been speculated that a gas cloud encountered by the jet in the inner $400 \mathrm{pc}$ of its journey out from the nucleus may have caused the bending of the inner jet structure (Schilizzi et al. 2001). On the one to few kpc scale, Hubble Space Telescope (HST) observations show a dust lane which wraps around the core, almost perpendicular to the jet axis (Martel et al. 1999; de Koff et al. 2000). Asymmetries and filamentary structures are seen in this dust lane suggesting that the structure is "dynamically young" (O'Dea et al. 2001). In addition, four young starforming regions along the dust lane have been found (see, e.g. O'Dea et al. 2001; Holt et al. 2007; Tremblay et al. 2010) giving further evidence for a recent interaction.

4C 31.04 is a compact radio source with two lobes separated by only about $100 \mathrm{pc}$ (e.g. Cotton et al. 1995; Giovannini et al. 2001). The radio source is hosted by the giant elliptical MCG 5-8-18 which is the dominant member of a small group of galaxies (Perlman et al. 2001). A central disk, elongated by about $1^{\prime \prime}(=1.2 \mathrm{kpc})$ roughly perpendicular to the jet axis, is visible in HST images (Perlman et al. 2001). Molecular observations by García-Burillo et al. (2007) show a gas disk ( 2 kpc in diameter) rotating along the same axis as the dust disk observed with the HST. However, the detected $\mathrm{HCO}^{+}$emission thickens along the minor axis, which García-Burillo et al. (2007) interprets as evidence for non-circular motions. Hence, the kpc scale disk structure of 4C 31.04 is not a dynamically relaxed system.

3C 236 and 4C 31.04 both have broad velocity $\mathrm{H}$ i absorption features $\left(\Delta v>300 \mathrm{~km} \mathrm{~s}^{-1}\right.$; van Gorkom et al. 1989; Mirabel 1990; Morganti et al. 2005b; Gupta et al. 2006) showing that large amounts of cold gas are present in their circumnuclear environments. In terms of the presence of a possible circumnuclear

\footnotetext{
1 The VLBA is operated by the National Radio Astronomy Observatory which is a facility of the National Science Foundation operated under co-operative agreement by Associated Universities, Inc.
}

cold gas (torus) structure it is interesting to note that the X-ray absorbing column densities detected against the cores of both sources are relatively low, $N_{\mathrm{H}}=1.3 \times 10^{20}$ atoms $\mathrm{cm}^{-2}$ and $N_{\mathrm{H}}=$ $5.8 \times 10^{20}$ atoms $\mathrm{cm}^{-2}$ for 3C 236 and 4C 31.04 respectively (Hardcastle \& Worrall 1999; Canosa et al. 1999). In other powerful radio galaxies much higher hydrogen column densities have been observed (with $N_{\mathrm{H}}>10^{23}$ atoms $\mathrm{cm}^{-2}$, e.g. Momjian et al. 2003; Morganti et al. 2004).

Estimates of the systemic velocity of 3C 236 yield a large spread ranging from $(29667 \pm 35) \mathrm{km} \mathrm{s}^{-1}$ (Fouque et al. 1992) to $(30150 \pm 150) \mathrm{km} \mathrm{s}^{-1}$ (Hill et al. 1996). The likely most reliable estimate, based on fits of the [O III] and $\mathrm{H} \beta$ emission lines in high resolution optical spectra (Holt, priv. comm.) gives, $v_{\text {sys }}=$ $29845 \mathrm{~km} \mathrm{~s}^{-1}$. Optical spectroscopy of 4C 31.04 (Marcha et al. 1996) yields a systemic velocity of $v_{\mathrm{sys}}=18002 \mathrm{~km} \mathrm{~s}^{-1}$, which is similar to the estimate of García-Burillo et al. (2007, $v_{\text {sys }}=$ $18057 \mathrm{~km} \mathrm{~s}^{-1}$ ) based on their mm-wave $\mathrm{HCO}^{+}$observations. In both sources the $\mathrm{H}_{\mathrm{I}}$ absorption straddles the systemic velocity of the systems (see van Gorkom et al. 1989).

\section{Observations}

Observations of 3C 236 and 4C 31.04 were performed on May 8th 1995 and July 7th 1995 respectively, using the ten stations of the VLBA plus a single antenna of the VLA. Two IFs (left and right circular polarisation) with a bandwidth of $4 \mathrm{MHz}$ and 128 spectral channels were centered at the frequency of the previously observed $\mathrm{H}$ I absorption $(\sim 1290 \mathrm{MHz}$ and $\sim 1340 \mathrm{MHz}$, for 3C 236 and 4C 31.04 respectively; van Gorkom et al. 1989; Mirabel 1990). A number of bright compact sources were observed as fringe finders and for bandpass calibration. The data were correlated in Soccoro, USA. A standard data reduction using AIPS was performed including fringe fitting, calibration and flagging of the data. A summary of the observations is given in Table 1 .

Initial continuum images (using only line-free channels) of both sources were made via iterative phase self-calibration/ deconvolution. A couple of cycles of amplitude and phase selfcalibration were performed. The resulting high resolution continuum maps look very similar to the maps obtained with dedicated continuum observations of 3C 236 (e.g. Schilizzi et al. 2001) and 4C 31.04 (e.g. Giroletti et al. 2003). However, the highest resolution images/cubes are not suitable for studying the $\mathrm{H}_{\mathrm{I}}$ absorption, due to a lack of sensitivity. Extensive experiments were carried out to determine the optimum $u v$ weighting in order to find the best combination of sensitivity and resolution for the spectral-line data. The best compromises between resolution and sensitivity were given by natural weighted cubes made using maximum uv-ranges of $10 M \lambda$ and $39 M \lambda$ for 3C 236 and 4C 31.04 respectively. The resulting cubes have beam $F W H M$ of 20 mas $\times 20$ mas and 12.1 mas $\times 8.7$ mas $(\mathrm{PA}=6.1)$ respectively. These data cubes were used for the analysis together with the corresponding continuum images. The off-source rms of all the data cubes and continuum images are dominated by Gaussian noise, however small calibration artifacts are visible to the eye at low levels in all four data products. The velocity resolution of the data cubes is $8.0 \mathrm{~km} \mathrm{~s}^{-1}$ (3C 236) and $7.4 \mathrm{~km} \mathrm{~s}^{-1}$ (4C 31.04) and the noise in the final line cube is $\sigma_{\mathrm{rms}}=0.4 \mathrm{mJy} \mathrm{beam}^{-1}$ per channel (3C 236) and $\sigma_{\text {rms }}=0.8 \mathrm{mJy} \mathrm{beam}^{-1}$ per channel (4C 31.04) (see Table 1 for details). 
Table 1. Summary of observations.

\begin{tabular}{|c|c|c|}
\hline & 3C 236 & 4C 31.04 \\
\hline Date of observation & 08 May 1995 & 07 July 1995 \\
\hline Bandwidth & $4 \mathrm{MHz}$ & $4 \mathrm{MHz}$ \\
\hline Number of spectral channels & 128 & 128 \\
\hline Channel separation & $8.0 \mathrm{~km} \mathrm{~s}^{-1}$ & $7.4 \mathrm{~km} \mathrm{~s}^{-1}$ \\
\hline Bandpass/flux calibrators & $3 \mathrm{C} 84,3 \mathrm{C} 273$ & $3 \mathrm{C} 84$ \\
\hline Weighting scheme & natural & natural \\
\hline Used UV-range & $<1.0 \times 10^{4} \mathrm{k} \lambda$ & $<3.9 \times 10^{4} \mathrm{k} \lambda$ \\
\hline Resolution & 20.0 mas $\times 20.0$ mas & $12.1 \mathrm{mas} \times 8.7 \mathrm{mas}$ \\
\hline Beam position angle & - & 6.1 \\
\hline rms noise $\left(\mathrm{mJy}\right.$ beam $\left.^{-1}\right)$ in the cubes & 0.4 & 0.8 \\
\hline velocity coverage in final cubes & $\sim 880 \mathrm{~km} \mathrm{~s}^{-1}$ & $\sim 890 \mathrm{~km} \mathrm{~s}^{-1}$ \\
\hline rms noise $\left(\mathrm{mJy}\right.$ beam $\left.^{-1}\right)$ in continuum maps & 1.0 & 1.8 \\
\hline
\end{tabular}

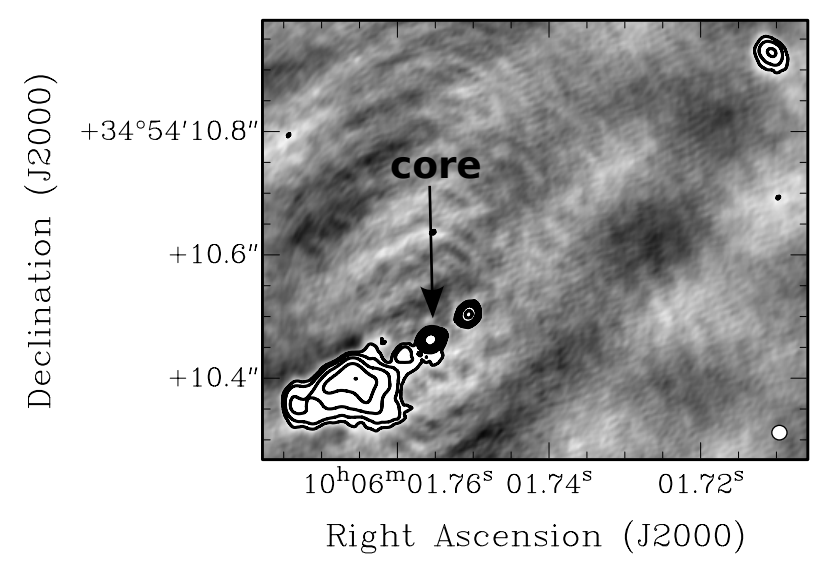

Fig. 1. 3C 236: continuum image at $1290 \mathrm{MHz}$. The lowest contour is at $5 \mathrm{mJy}$ beam $^{-1}$ with subsequent contours increasing by factors of 2 . The beam FWHM is indicated in the lower right corner.

\section{Results}

Broad absorption $\left(\Delta v \approx 350 \mathrm{~km} \mathrm{~s}^{-1}\right)$ is detected in both sources. The observational results for 3C 236 and 4C 31.04 are described in detail below.

\section{1. $3 C 236$}

\subsubsection{Continuum image}

The $1290 \mathrm{MHz}$ continuum image (Fig. 1) is very similar to the $1663 \mathrm{MHz}$ images shown by Barthel et al. (1985) and Schilizzi et al. (2001), except that our relatively narrow bandwidth observations do not allow us to properly image the faint emission surrounding the northwestern hot spot. We clearly detect (at the kpc scale) the core (labeled in Fig. 1), two knots to the northwest of the core and the southeastern jet/lobe structure. A comparison shows that we recover $\sim 60 \%(=1.36 \mathrm{Jy})$ of the flux density detected at $1290 \mathrm{MHz}$ in VLA observations by van Gorkom et al. (1989). The Mpc-scale structure - making 3C 236 one of the two largest single objects in the universe (e.g. Schilizzi et al. 2001; Machalski et al. 2008) - is not imaged in our VLBA observations.

\subsubsection{HI absorption}

An integrated absorption spectrum over the region where we detect absorption is shown in Fig. 2 (with the velocities shifted

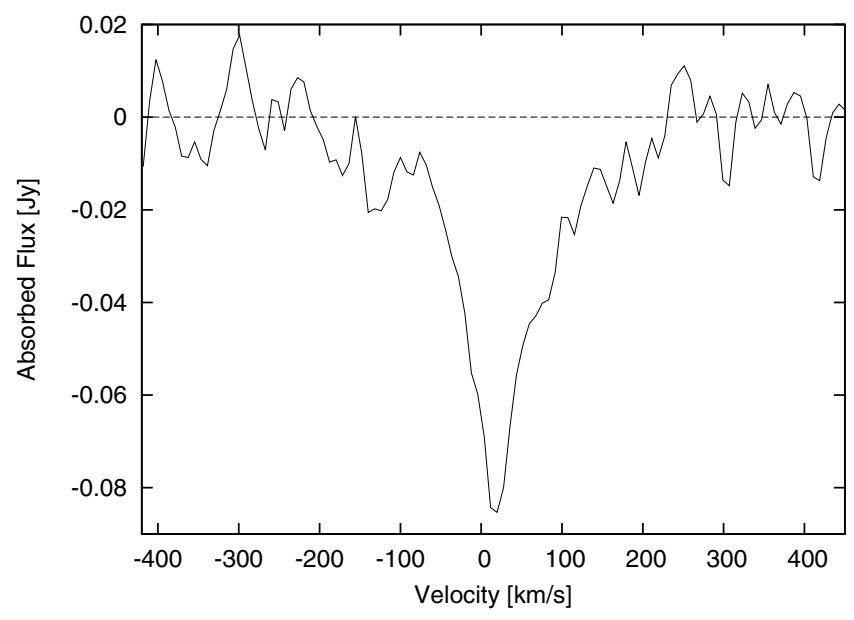

Fig. 2. 3C 236: integrated absorption spectrum over the region where absorption is detected (core and SE lobe) with the velocities shifted to the rest frame of the host galaxy.

to the rest frame of $3 \mathrm{C} 236$, see Sect. 5.1.2). Broad absorption $\left(\Delta v=359 \mathrm{~km} \mathrm{~s}^{-1}\right)$ is detected in the velocity range from $29628 \mathrm{~km} \mathrm{~s}^{-1}$ to $29987 \mathrm{~km} \mathrm{~s}^{-1}$ with the peak being located at $v=29840 \mathrm{~km} \mathrm{~s}^{-1}(z=0.09947)$.

The integrated spectrum looks very similar to the deep part of the absorption spectrum of broad band WSRT observations (Morganti et al. 2005b), including the flux scale within the uncertainties. However, we do not have the bandwidth to detect the faint, very broad $\Delta v \approx 1500 \mathrm{~km} \mathrm{~s}^{-1}$ outflow component (Morganti et al. 2005b) so that the absence of this broad component in the VLBA data is as expected.

Inspection of the data cube shows that the absorption is spatially resolved and is detected against the southeastern jet/lobe structure and the core. No absorption is detected against the northwestern continuum structures. The deepest absorption/largest opacity is detected against the tip of the southeastern jet structure and then decreases towards the core, as seen in the opacity map (Fig. 3, middle panel) and the opacity profile along PA $=117^{\circ}$ (Fig. 4). We note that weak absorption is even detected at distances slightly beyond the tip of the southeastern jet at 270 mas from the core, even though no underlying continuum could be detected. It remains unclear if this absorption is due to very high opacity absorption against weak continuum or is an imaging artefact.

An intriguing feature in the data is that a velocity centroid difference of approximately $40 \mathrm{~km} \mathrm{~s}^{-1}$ to $50 \mathrm{~km} \mathrm{~s}^{-1}$ is observed 


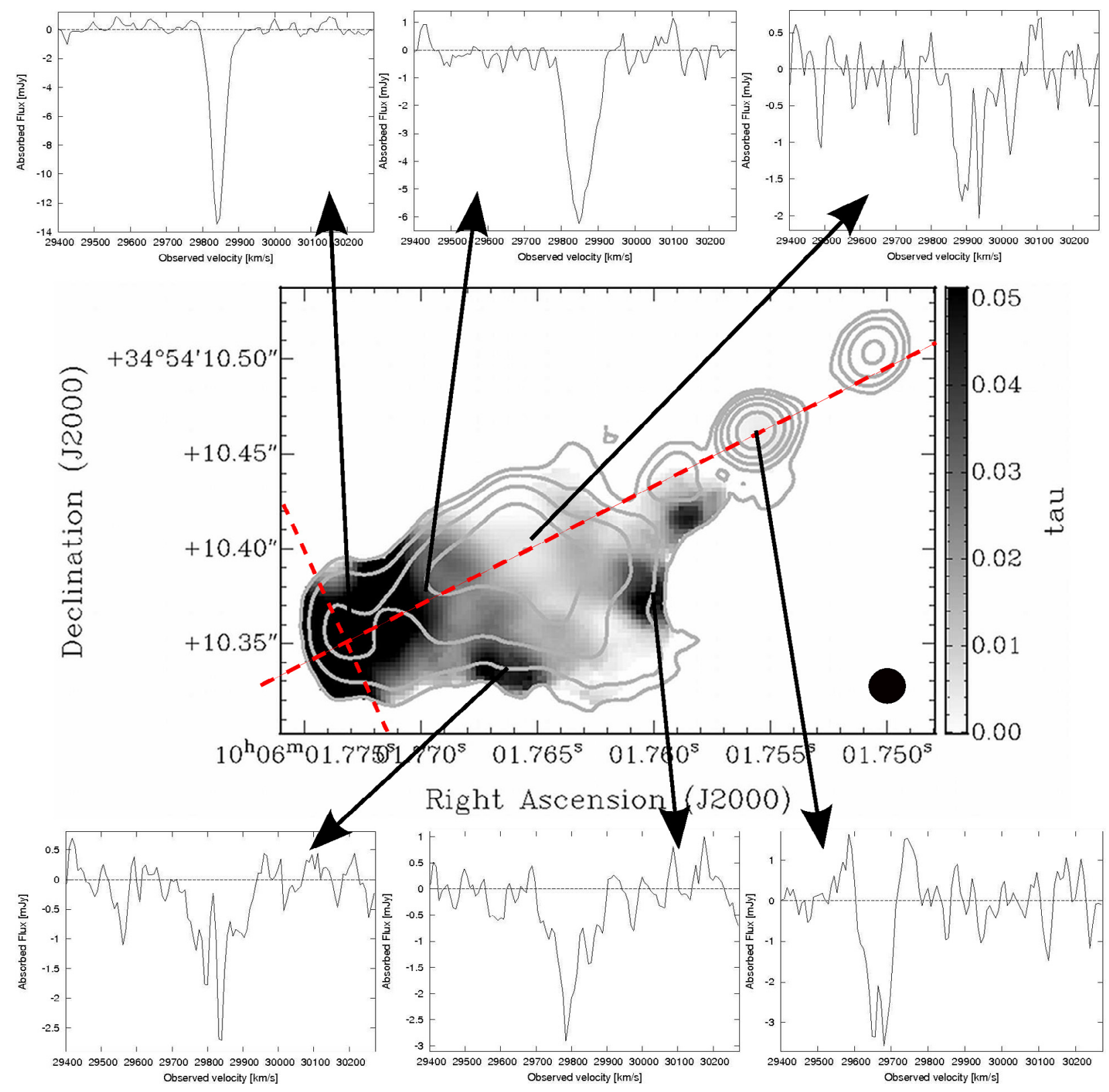

Fig. 3. 3C 236: middle panel: contours show continuum (same contour levels as Fig. 1). Grayscale shows the mean opacity over the rest frame velocity range $-192 \mathrm{~km} \mathrm{~s}^{-1}$ to $+167 \mathrm{~km} \mathrm{~s}^{-1}$. The beam FWHM is indicated in the lower right corner. The surrounding spectra show the absorption profiles at different locations indicated by the arrows. The dashed lines indicate the location of the two position-velocity slices presented in Fig. 5 .

perpendicular $\left(\mathrm{PA}=27^{\circ}\right)$ to the jet axis at the tip of the southeastern jet structure (Fig. 5, bottom panel). This gradient is seen over a spatial scale of $\approx 60$ mas (i.e. approx. 3 FWHM beams) and hence is well resolved.

At the tip of the southeastern jet structure (i.e. where the velocity gradient is observed), the velocity width is relatively narrow $\left(\Delta v \approx 100 \mathrm{~km} \mathrm{~s}^{-1}\right)$ but it increases as one moves closer to the core (e.g. at $r \approx 180$ mas). At smaller radii, the absorption becomes patchy (see Fig. 5, top panel). Interestingly, against the brightest part of the eastern jet continuum structure ( $r=140$ mas) virtually no absorption is detected (see top right panel of Fig. 3). However, three distinct regions of increased opacity are found at the southern edge of the southeastern jet structure. There is no direct connection (spatially or in velocity) between those "blobs". The nature of these clouds is discussed in Sect. 5.1.3.
The most blue-shifted absorption recovered by our observations is detected against the core at low opacity (Fig. 3, bottom right panel). This is interesting because of possible associations between this feature and the very broad $\left(\Delta v \approx 1500 \mathrm{~km} \mathrm{~s}^{-1}\right)$ out-flowing blue-shifted absorption detected by (Morganti et al. 2005b). In Sect. 5.1.3 we discuss this possibility further.

\section{2. $4 C 31.04$}

\subsubsection{Continuum image}

Figure 6 shows the $1340 \mathrm{MHz}$ continuum image of 4C 31.04. It appears very similar to the $1.7 \mathrm{GHz}$ image shown by Cotton et al. (1995) and the $5 \mathrm{GHz}$ (higher resolution) image from Giroletti et al. (2003). We detect the core and the western and eastern lobes. The peak flux density is $351 \mathrm{mJy} /$ beam and the total continuum flux recovered in our observations is $1.16 \mathrm{Jy}$. This is about $45 \%$ of the flux-density detected in VLA observations at 
C. Struve and J. E. Conway: The circumnuclear cold gas environments of the powerful radio galaxies 3C 236 and 4C 31.04
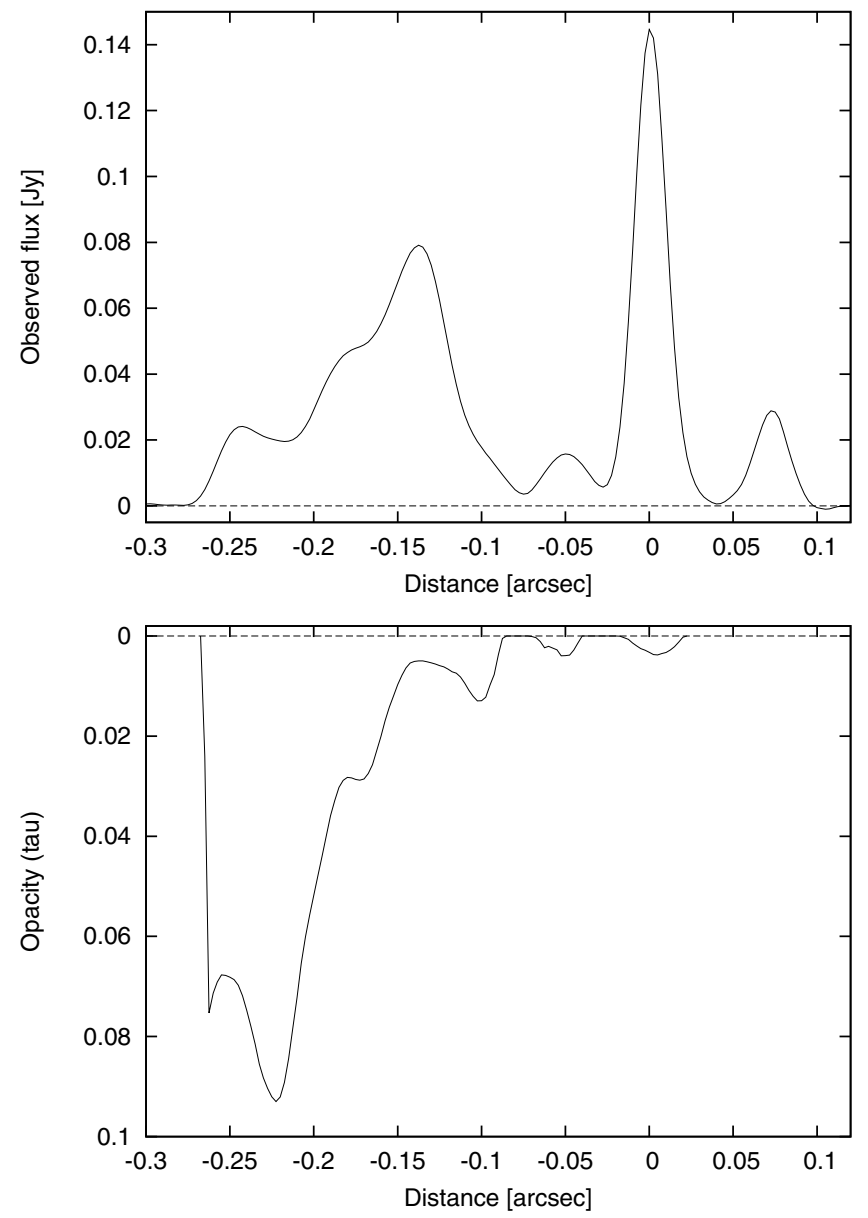

Fig. 4. 3C 236: top panel shows the continuum profile along a slice with $\mathrm{PA}=117^{\circ}$ (shown as long dashed line in Fig. 3). Negative offsets correspond to the SE (counter-) jet. Bottom panel: mean opacity profile averaged over the velocity width of the absorption $\left(\Delta v=359 \mathrm{~km} \mathrm{~s}^{-1}\right)$ along the same slice.

the same frequency (van Gorkom et al. 1989) and approximately $47 \%$ of the flux density detected at $1.7 \mathrm{GHz}$ (Cotton et al. 1995).

\subsubsection{HI absorption}

Absorption is detected against the core and both lobes. An integrated absorption spectrum over the entire region is shown in Fig. 7 (with the velocities shifted to the rest frame of 4C 31.04 , see Sect. 5.2.1). It shows a broad component $\left(\Delta v=337 \mathrm{~km} \mathrm{~s}^{-1}\right.$, from $17732 \mathrm{~km} \mathrm{~s}^{-1}$ to $18069 \mathrm{~km} \mathrm{~s}^{-1}$ ) and a narrow velocity component $\left(\Delta v=37 \mathrm{~km} \mathrm{~s}^{-1}\right)$. The peak of the broad component is located at $v=17906 \mathrm{~km} \mathrm{~s}^{-1}(z=0.05969)$ and the narrow component is redshifted relative to the broad component by $115 \mathrm{~km} \mathrm{~s}^{-1}\left(v_{\text {narrow }}=18121 \mathrm{~km} \mathrm{~s}^{-1}, z=0.06040\right)$. The integrated spectrum looks very similar to that seem in previous lower spatial resolution observations (Mirabel 1990; Gupta et al. 2006).

The opacity detected against the eastern lobe is approximately constant over the entire eastern lobe structure (Fig. 6). Gas with lower opacity is detected over a fraction of the western lobe lobe (see Fig. 6). In particular against the brightest continuum structure of the western lobe, no broad absorption component is detected. Overall, the absorption appears patchy (spatially and in velocity) on the western side.
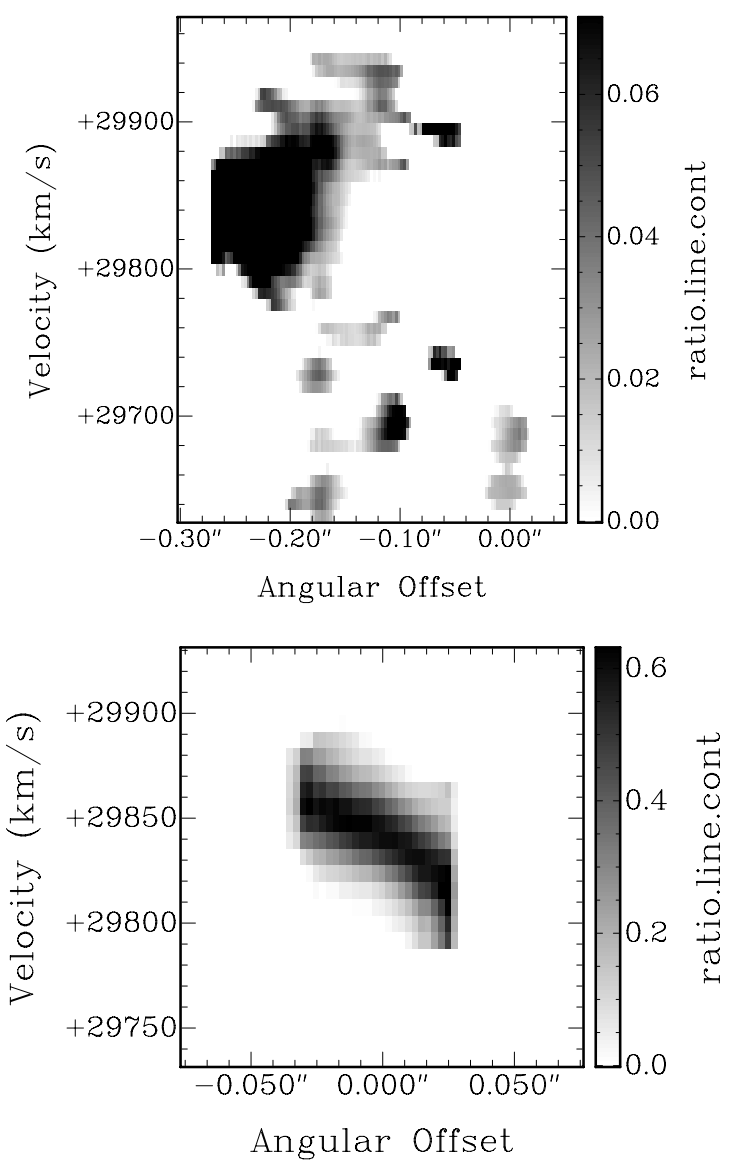

Fig. 5. 3C 236: position-velocity diagrams of the line-to-continuum ratio cube. Top panel: slice along PA $=117^{\circ}$ through the nucleus and through the peak at the southeastern lobe (as indicated by long dashed line in Fig. 3). Bottom panel: slice perpendicular to the slice in the top panel $\left(\mathrm{PA}=27^{\circ}\right)$, at the edge of the southeastern jet (as indicated by short dashed line in Fig. 3).

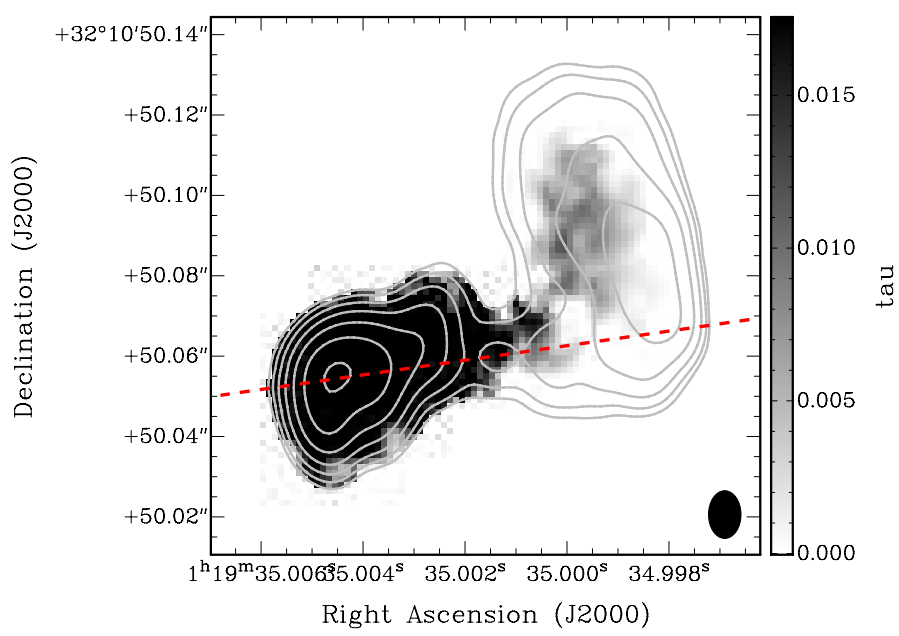

Fig. 6. 4C 31.04: contours show continuum, the lowest contour is at $5 \mathrm{mJy}$ beam ${ }^{-1}$ with subsequent contours increasing by factors of 2. Grayscale shows the mean opacity over the rest frame velocity range $-174 \mathrm{~km} \mathrm{~s}^{-1}$ to $+138 \mathrm{~km} \mathrm{~s}^{-1}$. The beam FWHM is indicated in the lower right corner. The dashed line indicates the location of the position-velocity slice presented in Fig. 8.

A position velocity slice (Fig. 8) along the jet axis (PA = $100^{\circ}$, slice indicated in Fig. 6) of the line-to-continuum cube 


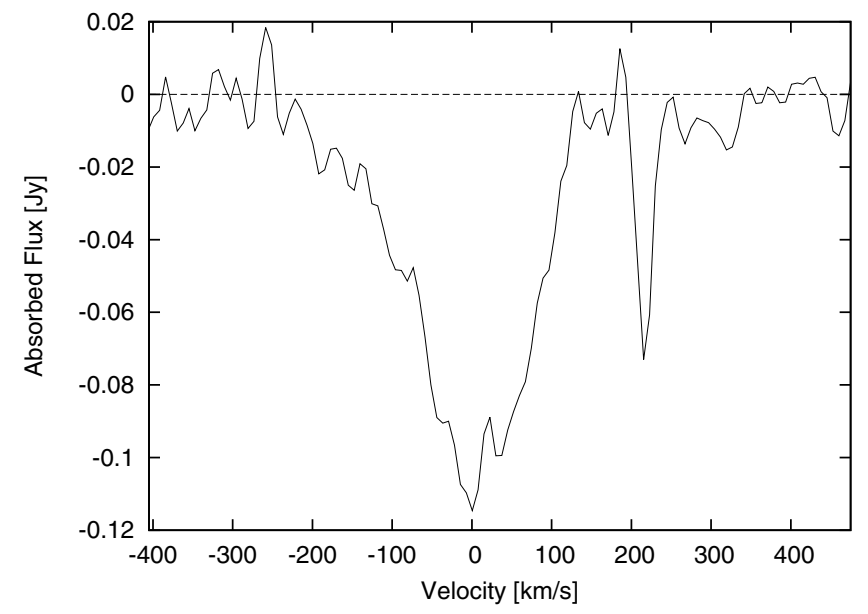

Fig. 7. 4C 31.04: integrated absorption spectrum over the region where absorption is detected (core, NW lobe and SE lobe) with the velocities shifted to the rest frame of the host galaxy (see Sect. 5.2.1).

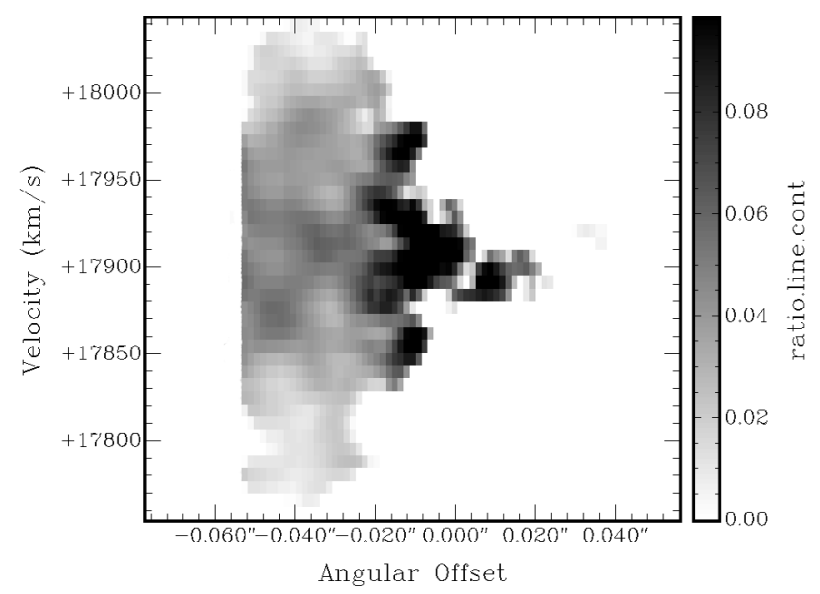

Fig. 8. 4C 31.04: position-velocity diagram of the line-to-continuum ratio cube along $\mathrm{PA}=100^{\circ}$ through the nucleus (as indicated in Fig. 6).

shows a broad velocity component at large distances from the core, 50 mas $>D>20$ mas. Closer to the nucleus, the velocity width decreases (at 20 mas $>D>0$ mas) and becomes relatively narrow at the core. The line-to-continuum ratio increases along the same direction such that the velocity integrated opacity stays approximately constant.

However, Fig. 8 is perhaps misleading in the sense that it does not reveal the sensitivity limitations of the observations. The fact that the velocity width decreases closer to the nucleus may solely be the result of insufficient sensitivity of the observations. Figure 9 shows along a slice the distribution of lineto-continuum ratio at the velocity channel with the deepest absorption $\left(v=17906 \mathrm{~km} \mathrm{~s}^{-1}\right)$ going through the nucleus and the eastern hot spot $\left(\mathrm{PA}=100^{\circ}\right)$. The solid line shows the observed ratio while the dashed line indicates the $3 \sigma$ detection limit. As is evident from the figure, the observations are only just sensitive enough to detect the peak of the spectrum at -10 mas $<D<0$ mas from the core against the eastern lobe. The radio continuum of the western lobe is too weak to detect absorption at the same opacity as detected against the eastern host spot. Lack of sensitivity is likely the reason why the apparent velocity width decreases going from east to west in Fig. 8. While the peak of the spectrum is still detectable over the eastern lobe, parts of the profile wings drop below the detection limit such that

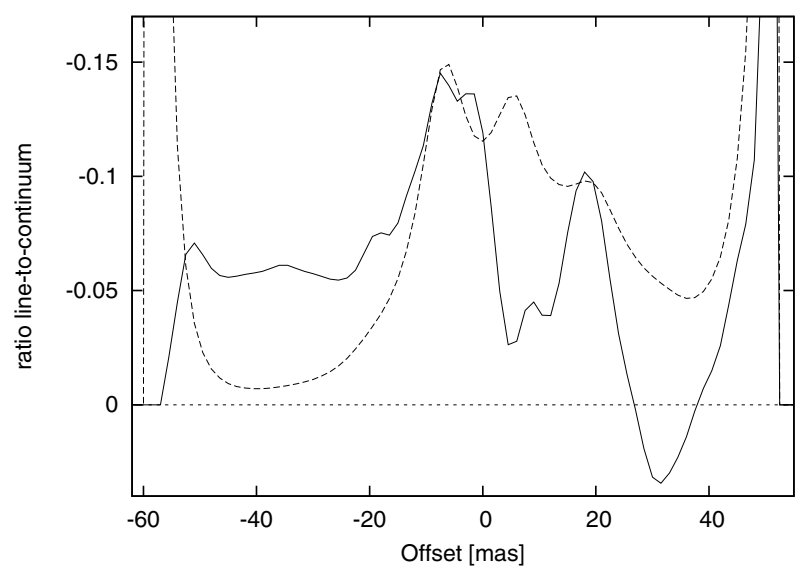

Fig. 9. 4C 31.04: solid line shows the observed line-to-continuum ratio at velocity $v=17906 \mathrm{~km} \mathrm{~s}^{-1}$ along a slice going through the nucleus and the eastern hot spot $\left(\mathrm{PA}=100^{\circ}\right.$, as indicated in Fig. 6). The dashed line shows the $3 \sigma$ detection limit on the line-to-continuum ratio.

the observed profile looks narrower -20 mas $<D<0$ mas from the core than it is probably the case.

No velocity gradient is detected along the jet and only a hint of a velocity gradient is detected perpendicular to the jet axis. The difficulty in setting strong constraints on this perpendicular component of the velocity gradient is not surprising, given that the continuum one beam north and south of the eastern hot spot (i.e. in the direction where a velocity gradient would be expected) is too weak to detect absorption with the same opacity as observed against the hot spot. However the orientation of the tentative velocity gradient we do see is consistent with the velocity gradient found in lower resolution $\mathrm{HCO}^{+}$observations (García-Burillo et al. 2007) on larger scales.

The redshifted, narrow absorption component is most prominently seen against the western lobe especially near its continuum peak. However, absorbing gas with the same velocity is also detected against a large fraction of the eastern lobe. The narrow absorption is not detected against the core, but this is expected given its low continuum brightness.

\section{Discussion}

In the coming two subsections we discuss the Hi absorbing structures of both sources individually. In both cases we argue that the simplest explanation is that the broad $\mathrm{H}$ I absorption lies in a flattened disk-like structure which forms a part/continuation of the sub-kpc scale disks previously observed in these sources. We discuss possible constraints on the gas properties within the postulated atomic disk structures.

\section{1. $3 C 236$}

\subsubsection{The absorbing $\mathrm{H}$ I structures}

Neutral hydrogen absorption is detected against the core (with low opacity) and against the southeastern jet/lobe, which is thought to be the jet pointing away from us (Schilizzi et al. 2001). The existence of a velocity gradient perpendicular to the projected counter-jet direction (Sect. 4.1.2) is then consistent with a foreground rotating $\mathrm{H}_{\mathrm{I}}$ absorbing disk/ring lying in a plane roughly perpendicular to the jet axis. Note however that given the small area over which absorption can be traced more complex kinematic interpretations involving non-circular motions of gas are can also fit the data. Providing support to the 
disk interpretation the orientation of the observed $\mathrm{H}_{\mathrm{I}}$ gradient $\left(\mathrm{PA}=27^{\circ}\right)$ is roughly the same as the disk found on HST images (see, e.g. O'Dea et al. 2001; Tremblay et al. 2010) on comparable scales. The absorbing gas at distances $>200$ mas from the core may therefore be part of the star-forming ring structure identified on the HST images. Circumnuclear $\mathrm{H}$ I absorbing disks oriented roughly perpendicular to the jet direction are not uncommon. In a few other sources similar absorbing circumnuclear structures have been detected e.g. in NGC 4261 (van Langevelde et al. 2000) and Cygnus A (Struve \& Conway 2010).

The presence of an $\mathrm{H}_{\mathrm{I}}$ absorbing disk/ring can however only explain part of the absorption in $3 \mathrm{C} 236$. At distances less than 200 mas from the core, the opacity rapidly drops and the absorption becomes patchy (Sect. 4.1.2). We have very few constraints on these individual H I clouds, however we will argue that these clouds are not part of the absorbing disk. It is possible instead that some of these clouds could be interacting with the jet (see Sect. 5.1.3).

\subsubsection{Properties of the HI disk}

Under the assumption that the absorption at the tip of the southeastern lobe structure is caused by a foreground gas disk which is part of the HST ring structure (e.g. O'Dea et al. 2001; Tremblay et al. 2010), we can derive some of its basic properties. While only a small part of the $\mathrm{H}$ I disk is detected in absorption it is possible to estimate the cold gas column density as well as constrain the disk kinematics.

The HST observations indicate a disk position angle of $\mathrm{PA}=$ $30^{\circ}$ (O'Dea et al. 2001). Adopting this orientation for our $\mathrm{H}$ I disk and assuming that the disk normal is approximately aligned with the jet axis (as found in many other radio galaxies), the disk inclination must be close to $i=60^{\circ}$ (see Schilizzi et al. 2001). The inner edge of the disk is defined by the peak in the line-to-continuum ratio ( $D=220$ mas; Fig. 4), which corresponds to a de-projected radius of 440 mas. Unfortunately, we have no constraints on the maximum radius to which the disk extends because we run out of continuum. Because absorption is even detected at slightly larger radii than we can image the underlying continuum (Sect. 4.1.2), the $\mathrm{H}_{\mathrm{I}}$ disk may extend to radii $r>500$ mas from the core.

Given the observed velocity gradient (which is perpendicular to the jet axis), we can also constrain the systemic velocity and the rotation velocity. Fixing the geometry of the disk to PA $=30^{\circ}$ and $i=60^{\circ}$ yields $v_{\text {sys }}=29820 \mathrm{~km} \mathrm{~s}^{-1}$ $(z=0.09940)$. Interestingly, our value for the systemic velocity is within $20 \mathrm{~km} \mathrm{~s}^{-1}$ of the peak of the absorption profile and agrees within the uncertainties $\left(\Delta v=25 \mathrm{~km} \mathrm{~s}^{-1}\right)$ with the value derived by Holt from fits of emission lines (Sect. 2). The estimated rotation velocity at $r=440$ mas $(=880 \mathrm{pc})$ is $170 \mathrm{~km} \mathrm{~s}^{-1}<v_{\text {rot }}<210 \mathrm{~km} \mathrm{~s}^{-1}$, assuming an observed gradient corresponding to a change of velocity over $\sim 60$ mas of between $40 \mathrm{~km} \mathrm{~s}^{-1}$ and $50 \mathrm{~km} \mathrm{~s}^{-1}$ (Sect. 4.1.2). The above range of rotation velocities implies an enclosed mass within $880 \mathrm{pc}$ radius of 5.9-9.0 $\times 10^{9} M_{\mathrm{o}}$. Although this mass estimate becomes quite uncertain if we also include uncertainties in disk inclination it is a reasonable value for the enclosed mass on sub-kpc scales; supporting the disk interpretation as the origin of the $\mathrm{HI}$ absorption at the tip of the SE jet.

Besides the kinematical information, we can also estimate the peak Hi column density from the opacity map (Fig. 3) to be $N_{\mathrm{HI}} T_{\mathrm{spin}}^{-1}=6.1 \times 10^{19} \mathrm{~cm}^{-2}$. Assuming a spin temperature of $100 \mathrm{~K}$, the column density is $N_{\mathrm{HI}}=6.1 \times 10^{21} \mathrm{~cm}^{-2}$. This is similar to what has been detected e.g. in Centaurus A in emission at slightly larger distances from the core (Struve et al. 2010). In 3C 236 the $\mathrm{H}$ I column density rapidly drops towards the core and is $N_{\mathrm{HI}}=2.1 \times 10^{20} \mathrm{~cm}^{-2}$ against the nucleus. This is slightly higher than the measured X-ray column density $\left(N_{\mathrm{H}}=1.3 \times\right.$ $10^{20} \mathrm{~cm}^{-2}$ Hardcastle \& Worrall 1999).

\subsubsection{Interpretation of absorption features along jet and against core.}

Assuming the jet and foreground $\mathrm{H}_{\mathrm{I}}$ absorbing disk geometry given in Sect 5.1.2, as required to explain the perpendicular velocity gradient against the tip of the SE jet, then no interaction between the jet and the foreground gas disk is expected. This interpretation also implies that the southeastern jet/lobe structure is not confined by the gas disk. It is therefore only a coincidence that the size of the southeastern lobe is approximately the same as the radius of the star-forming ring structure. Other H I absorption features in our data are however difficult to explain in terms of a $\mathrm{H}_{\mathrm{I}}$ disk. Several discrete $\mathrm{H}$ i absorbing clouds are observed at distances $<200$ mas from the core (Sect. 4.1.2 and Fig. 5, top panel). These clouds do not form a coherent structure in either morphology or kinematics. It seems unlikely therefore that these clouds are part of the cold gas disk but instead lie above or below the disk plane.

The three clouds with highest opacity are all located at the southern edge of the southeastern lobe structure (see Fig. 3) exactly along the jet axis of the Mpc-scale structure (Barthel et al. 1985), which is misaligned with respect to the kpc-scale jet structure by $\sim 7^{\circ}$ (O'Dea et al. 2001). The location of these three clouds - though only seen in projection - together with the lack of a coherent velocity structure, suggests that these clouds might be interacting with the jet. We speculate that these clouds might even be blocking the expansion of the jet along the PA of the large-scale jet and causing the misalignment between the inner and outer jet structure. The velocities of all three clouds are close to systemic though on average slightly redshifted $\left(-25 \mathrm{~km} \mathrm{~s}^{-1}<v_{\text {clouds }}-v_{\text {sys }}<75 \mathrm{~km} \mathrm{~s}^{-1}\right)$. Since the southeastern lobe is likely pointing away from us then these clouds are on average moving (relatively slowly) away from the nucleus.

In addition to the three clouds at the southern edge of the lobe structure a number of other $\mathrm{H}_{\mathrm{I}}$ clouds are tentatively detected against the jet with low opacity. Some of those clouds are only detected against bright parts of the continuum. In particular the broad absorption profiles detected at $D \approx 180$ mas from the core (Sect. 4.1.2) may be due to the superposition of cloud and disk features. At this position the lower velocity part of the broad component fits the velocities seen at larger radii from the nucleus (which can be explained by a rotating disk structure) while the higher velocity part has the same velocity as the bordering narrow absorption component located towards the core direction. The nature/origin of these additional $\mathrm{H}_{\mathrm{I}}$ absorbing clouds (and whether they also may interact with the jet) is unclear. In any case the presence of such absorption features shows that a number of unsettled gas clouds are likely located in the few hundred parsec vicinity of the nucleus.

An intriguing question is the location of the fast neutral hydrogen outflow detected by Morganti et al. (2005b). We do not have the bandwidth to detect this very broad absorption feature but some narrower blue-shifted absorption is detected. In other sources with a detected outflow, the location of the outflow is offnucleus. In 3C 305 and IC 5063, for instance, the H I outflows are located $0.5 \mathrm{kpc}$ and $1.6 \mathrm{kpc}$ from the core (Morganti et al. 2005a, 2007). In our observations blueshifted absorption is detected 
against the core but also weakly against the southeastern lobe 200 mas (=400 pc) from the core (see Fig. 5). Whether either of these two places is the location of the fast outflow component is not clear, new sensitive broad band VLBI observations are required to locate the outflow in $3 \mathrm{C} 236$.

\section{2. $4 C 31.04$}

\subsubsection{The broad velocity component}

The broad velocity component detected against the eastern hot spot together with the tentative velocity gradient (which is consistent with the gradient found in lower resolution $\mathrm{HCO}^{+}$observations; García-Burillo et al. 2007) suggest that an $\mathrm{H}$ I absorbing circumnuclear disk may also exist in 4C 31.04 with a PA $\approx 10^{\circ}$. Unfortunately, the limitations of the observations make it impossible to rule out other explanations like e.g. non-circular motions. However, evidence for the existence of circumnuclear disks has been found in a few sources, including e.g. 3C 236 (Sect. 5.1), NGC 4261 (van Langevelde et al. 2000), 1946+708 (Peck \& Taylor 2001) and Cygnus A (Struve \& Conway 2010). In all such sources, the velocity dispersion is of similar order to the $\sim 70 \mathrm{~km} \mathrm{~s}^{-1}$ found for $4 \mathrm{C} 31.04$.

Due to the limitations of the observations it is impossible to derive the exact radius, rotation velocity or scale-height of the absorbing disk - assuming that the interpretation of a circumnuclear disk is correct. While the observed absorption profile apparently becomes narrower towards the core (compared to the velocity width radius at the eastern hot spot) this is likely the result of insufficient sensitivity (Sect. 4.2.2). In fact, for a circumnuclear gas disk of roughly constant opening angle one would expect that the velocity profile width should increases toward the nucleus as the rotational velocity increases. Whether this is indeed the case can only be investigated with more sensitive VLBI observations.

Despite the sparsity of information some rough estimates of the basic properties of a possible absorbing $\mathrm{H}_{\mathrm{I}}$ disk can still be made. If the absorption is indeed caused by a gas disk, the peak absorption against the core must indicate the systemic velocity, which is $v_{\text {sys }}=17906 \mathrm{~km} \mathrm{~s}^{-1}$. We note that the velocity of the deepest absorption against the core coincides with the velocity of peak opacity in the integrated spectrum. This velocity is slightly lower than the systemic velocity obtained from $\mathrm{HCO}^{+}$observations $\left(v_{\text {sys }}=18057 \mathrm{~km} \mathrm{~s}^{-1}\right.$; García-Burillo et al. 2007, see also Sect. 2). However, García-Burillo et al. (2007) noted that the rotating molecular disk (with size 1.'6) is not dynamically relaxed. Since their resolution is $\sim 1^{\prime \prime}$, unsettled gas may have erroneously been classified as gas located in the disk, resulting in a slightly too high estimate for $v_{\text {sys }}$.

Under the assumption that the Hi disk PA agrees with the disk orientation seen on larger scales in $\mathrm{HCO}^{+}$and in HST images $\left(\mathrm{PA} \approx 10^{\circ}\right.$; García-Burillo et al. 2007; Perlman et al. 2001) we can estimate the radius of the disk assuming an inclination. According to Giroletti et al. (2003), the lobes are oriented $>75^{\circ}$ from the line-of-sight, which implies that the disk is likely at $i \geq 75^{\circ}$. This implies that the $\mathrm{H}$ I absorbing gas detected against the eastern hot spot is located at $r \geq 200 \mathrm{pc}$. Assuming in addition that the opening angle of the disk is $14^{\circ}$ (i.e. the same as found in other sources, see, e.g. van Langevelde et al. 2000; Struve \& Conway 2010), the scaleheight is $\Delta h \approx 50 \mathrm{pc}$ and the rotation amplitude should be of order $v_{\text {rot }} \approx 280 \mathrm{~km} \mathrm{~s}^{-1}$. It is possible that the $\mathrm{H}_{\mathrm{I}}$ disk extends to radii $r<200 \mathrm{pc}$, but the lack of sensitivity makes it impossible to determine how far in. Additional complications are the uncertainty in inclination and scale-height, whose extent could explain the absorption detected close to the nucleus and against the western lobe. The uncertainties in radius, inclination and spin temperature make it also impossible to derive a meaningful enclosed mass estimate for any putative atomic gas disk, therefore, a comparison with the molecular gas mass estimate of García-Burillo et al. (2007) cannot be made.

As a final property of the atomic hydrogen in 4C 31.04 we can estimate the observed H I column density which is in the relativly narrow range $N_{\mathrm{HI}}=1.2 \times 10^{19} T_{\text {spin }}$ atoms cm $\mathrm{cm}^{-2}$ (seen against the core) to $2.4 \times 10^{19}$ atoms $\mathrm{cm}^{-2}$ (detected against the southeastern jet). For a typical spin temperature of $100 \mathrm{~K}$, this translates into $N_{\mathrm{HI}}=1.2-2.4 \times 10^{21}$ atoms $\mathrm{cm}^{-2}$. However, the column density towards the core may be underestimated if large fractions of the wings of the absorption profile are lost in the noise (due to sensitivity limitations). It may therefore be that the $\mathrm{H}$ I column increases rather than decreases towards the core. It is interesting to note that the ROSAT X-ray column (Canosa et al. 1999 ) is already a factor of 2-4 lower than what is observed for $N_{\mathrm{HI}}$ toward the core. This could possibly be explained if, as expected, the X-ray core is much smaller than the radio core and the atomic gas is located in clouds which do not cover the $\mathrm{X}$-ray core.

\subsubsection{The narrow velocity component}

While we argue that the broad absorption component in 4C 31.04 is caused by gas located in a rotating disk structure, the narrow absorption component is likely to have a different origin. This narrow velocity component is redshifted by $115 \mathrm{~km} \mathrm{~s}^{-1}$ with respect to systemic implying foreground gas moving towards the nuclear region. Most of the narrow absorption component is detected against the northwestern lobe, but absorption at the same velocity is also observed against the southeastern lobe structure. In total, the narrow absorption component covers a region of almost 120 pc across both lobes. Since the velocity width is small $\left(F W H M=23 \mathrm{~km} \mathrm{~s}^{-1}\right)$ and is seen against both lobes, we suspect that this structure is relatively far away from the core $(\gg 100 \mathrm{pc})$ as otherwise tidal forces would widen the velocity width and introduce velocity centroid variations (which are not observed). Hence, though moving towards the core, this narrow velocity width gas component is not (yet) providing the fuel for the AGN.

The origin and structure of the narrow absorption feature is unclear but may be related to the fact that 4C 31.04 is not a dynamically relaxed system. The molecular gas disk (with $r \sim$ $1 \mathrm{kpc}$ ) is not completely settled (García-Burillo et al. 2007) and Perlman et al. (2001) speculate about a recent merger or accretion event based on features seen in HST colour maps. 4C 31.04 is located in the center of a small galaxy group, surrounded by several fainter companions (Perlman et al. 2001) and hence the narrow absorption component may be a sign of the aftermath of a recent disturbance caused by one of the companions.

\section{Summary}

We have reported VLBA H I absorption observations of the circumnuclear environments of the powerful radio galaxies 3C 236 and 4C 31.04. In both sources the broad absorption components $\left(\Delta v \approx 350 \mathrm{~km} \mathrm{~s}^{-1}\right)$ are spatially resolved and detected against the counter-jets and the cores. In 4C 31.04 weaker broad absorption is also detected against the jet. A velocity gradient roughly perpendicular to the jet axis is observed in 3C 236, indications of a similar gradient are also present in $4 \mathrm{C} 31.04$. 
The data are consistent with the presence of $\mathrm{H}$ i absorbing disks with $r \approx 900 \mathrm{pc}$ and $r \geq 200 \mathrm{pc}$ for 3C 236 and 4C 31.04 respectively although alternative explanations cannot be ruled out. Absorbing disks with similar properties have been found in a few other sources, though typically on scales below $\sim 100 \mathrm{pc}$ radius (e.g. in Centaurus A and Cygnus A; Morganti et al. 2008; Struve \& Conway 2010).

In addition to the absorbing disk, cold gas closer to the nucleus seems to be present in $3 \mathrm{C} 236$. A few $\mathrm{H}$ i clouds may be interacting with the counter-jet, possibly explaining why the inner $\mathrm{kpc}$ scale jet structure is misaligned to the Mpc scale structure by $7^{\circ}$. These possibly intervening clouds are located (in projection) $\sim 120$ pc to $\sim 370$ pc from the nucleus and have velocities close to the systemic velocity. At least some of these clouds may have an outflowing velocity component. No direct evidence is found that cold gas is fuelling the AGN in 3C 236. Likewise do we find no evidence that cold gas is fuelling the AGN of $4 \mathrm{C} 31.04$. The broad absorption component in this source is most naturally explained by a disk structure with $r \geq 200 \mathrm{pc}$ (although the disk could also extend to smaller radii) and the gas structure causing the narrow, redshifted absorption component is likely much further than $100 \mathrm{pc}$ from the black hole. This redshifted component may show the aftermath of a recent accretion/ merging event.

Our results of 3C 236 and 4C 31.04 show - in agreement with previous studies at other wavelengths - that both systems are not dynamically relaxed systems and that unsettled gas structures exist. However, in both sources the $\mathrm{H}_{\mathrm{I}}$ absorbing clouds are not close enough to the black hole to directly cause the triggering of the AGN.

High resolution observations of molecules are desirable to probe the physical properties close to the AGN and to better constrain the kinematics in the circumnuclear environment, to find out about the neutral gas density and its radial dependence, and to locate the origin of the (neutral) gas outflow. In addition, such observations should allow us to study more thoroughly the possible interaction of gas clouds with the jet in 3C 236.

Acknowledgements. We thank the anonymous referee for his/her comments that helped to improve the paper. C.S. thanks The Netherlands Organisation for Scientific Research (NWO) for funding this project under Rubicon grant 680-501009. J.C. acknowledges financial support from the Swedish Science Research Council.

\section{References}

Barthel, P. D., Miley, G. K., Jagers, W. J., Schilizzi, R. T., \& Strom, R. G. 1985, A\&A, 148, 243

Canosa, C. M., Worrall, D. M., Hardcastle, M. J., \& Birkinshaw, M. 1999, MNRAS, 310,30

Combes, F., Young, L. M., \& Bureau, M. 2007, MNRAS, 377, 1795

Conway, J. E. 1996, in Extragalactic Radio Sources, eds. R. D. Ekers, C. Fanti, \& L. Padrielli, IAU Symp., 175, 92

Conway, J. E. 1997, in Atomic hydrogen absorption in radio-loud sources, eds. N. Jackson, \& R. J. Davis, 153
Conway, J. E., \& Schilizzi, R. T. 2000, in EVN Symposium 2000, Proc. of the 5th Europ. VLBI Network Symp., eds. J. E. Conway, A. G. Polatidis, R. S. Booth, \& Y. M. Pihlström, 123

Cotton, W. D., Feretti, L., Giovannini, G., et al. 1995, ApJ, 452, 605

de Koff, S., Best, P., Baum, S. A., et al. 2000, ApJS, 129, 33

Emonts, B. H. C., Morganti, R., Struve, C., et al. 2010, MNRAS, 406, 987

Fouque, P., Durand, N., Bottinelli, L., Gouguenheim, L., \& Paturel, G. 1992, in Catalogue of Optical Radial Velocities (Paris: Obs. Lyon, Paris-Meudon)

García-Burillo, S., Combes, F., Neri, R., et al. 2007, A\&A, 468, L71

Giovannini, G., Cotton, W. D., Feretti, L., Lara, L., \& Venturi, T. 2001, ApJ, 552, 508

Giroletti, M., Giovannini, G., Taylor, G. B., et al. 2003, A\&A, 399, 889

Gupta, N., Salter, C. J., Saikia, D. J., Ghosh, T., \& Jeyakumar, S. 2006, MNRAS, 373,972

Hardcastle, M. J., \& Worrall, D. M. 1999, MNRAS, 309, 969

Hicks, E. K. S., Davies, R. I., Malkan, M. A., et al. 2009, ApJ, 696, 448

Hill, G. J., Goodrich, R. W., \& Depoy, D. L. 1996, ApJ, 462, 163

Holt, J., Tadhunter, C. N., González Delgado, R. M., et al. 2007, MNRAS, 381, 611

Jaffe, W., Meisenheimer, K., Röttgering, H. J. A., et al. 2004, Nature, 429, 47

Machalski, J., Kozieł-Wierzbowska, D., Jamrozy, M., \& Saikia, D. J. 2008, ApJ, 679,149

Magorrian, J., Tremaine, S., Richstone, D., et al. 1998, AJ, 115, 2285

Marcha, M. J. M., Browne, I. W. A., Impey, C. D., \& Smith, P. S. 1996, MNRAS, 281,425

Marconi, A., Risaliti, G., Gilli, R., et al. 2004, MNRAS, 351, 169

Martel, A. R., Baum, S. A., Sparks, W. B., et al. 1999, ApJS, 122, 81

Merloni, A., \& Heinz, S. 2008, MNRAS, 388, 1011

Mirabel, I. F. 1990, ApJ, 352, L37

Momjian, E., Romney, J. D., Carilli, C. L., Troland, T. H., \& Taylor, G. B. 2003, ApJ, 587, 160

Morganti, R., Oosterloo, T. A., Tadhunter, C. N., et al. 2004, A\&A, 424, 119

Morganti, R., Oosterloo, T. A., Tadhunter, C. N., van Moorsel, G., \& Emonts, B. 2005a, A\&A, 439, 521

Morganti, R., Tadhunter, C. N., \& Oosterloo, T. A. 2005b, A\&A, 444, L9

Morganti, R., de Zeeuw, P. T., Oosterloo, T. A., et al. 2006, MNRAS, 371, 157

Morganti, R., Holt, J., Saripalli, L., Oosterloo, T. A., \& Tadhunter, C. N. 2007, A\&A, 476, 735

Morganti, R., Oosterloo, T., Struve, C., \& Saripalli, L. 2008, A\&A, 485, L5

Nenkova, M., Sirocky, M. M., Nikutta, R., Ivezić, Ž., \& Elitzur, M. 2008, ApJ, 685,160

Ocaña Flaquer, B., Leon, S., Combes, F., \& Lim, J. 2010, A\&A, 518, A9

O'Dea, C. P., Koekemoer, A. M., Baum, S. A., et al. 2001, AJ, 121, 1915

Oosterloo, T. A., Morganti, R., Sadler, E. M., van der Hulst, T., \& Serra, P. 2007, A\&A, 465, 787

Oosterloo, T., Morganti, R., Crocker, A., et al. 2010, MNRAS, 409, 500

Peck, A. B., \& Taylor, G. B. 2001, ApJ, 554, L147

Perlman, E. S., Stocke, J. T., Conway, J., \& Reynolds, C. 2001, AJ, 122, 536

Rees, M. J. 1984, ARA\&A, 22, 471

Schilizzi, R. T., Tian, W. W., Conway, J. E., et al. 2001, A\&A, 368, 398

Schinnerer, E., Eckart, A., Tacconi, L. J., Genzel, R., \& Downes, D. 2000, ApJ, 533,850

Shankar, F., Salucci, P., Granato, G. L., De Zotti, G., \& Danese, L. 2004, MNRAS, 354, 1020

Soltan, A. 1982, MNRAS, 200, 115

Struve, C., \& Conway, J. E. 2010, A\&A, 513, A10

Struve, C., Oosterloo, T. A., Morganti, R., \& Saripalli, L. 2010, A\&A, 515, A67

Tremblay, G. R., O’Dea, C. P., Baum, S. A., et al. 2010, ApJ, 715, 172

Tristram, K. R. W., Raban, D., Meisenheimer, K., et al. 2009, A\&A, 502, 67

van der Wolk, G., Barthel, P. D., Peletier, R. F., \& Pel, J. W. 2010, A\&A, 511, A64

van Gorkom, J. H., Knapp, G. R., Ekers, R. D., et al. 1989, AJ, 97, 708

van Langevelde, H. J., Pihlström, Y. M., Conway, J. E., Jaffe, W., \& Schilizzi, R. T. 2000, A\&A, 354, L45 